\title{
Towards an automated generator of urban building energy loads from 3D building models
}

\author{
Alessandro Maccarini ${ }^{1} \quad$ Michael Mans $^{2} \quad$ Christian G. Sørensen $^{1} \quad$ Alireza Afshari $^{1}$ \\ ${ }^{1}$ Department of the Built Environment, Aalborg University, Denmark, amac@build. aau.dk \\ ${ }^{2}$ Institute for Energy Efficient Buildings and Indoor Climate, RWTH Aachen University, Germany
}

\begin{abstract}
Buildings in cities are one of the major contributors of carbon emissions worldwide. Thus, improving building energy efficiency is one of the key strategies towards sustainable urbanization. Urban building energy modeling (UBEM) is a valuable methodology to tackle these challenges, as it provides users with the energy demand of the building stock, scenarios evaluation, peak loads and other useful analyses. This paper presents an open-source tool to automatically convert 3D building models into ready-to-run Modelica models for urban energy simulations. The software enables users to create 3D building geometries, perform data enrichment and execute model generation of reduced order Modelica models. The software is written in Python and it has been developed as an add-on for the 3D creation application Blender. The first part of the paper describes the general approach and the architecture of the tool. In the second part, a demonstration of the tool's capabilities is illustrated.
\end{abstract}

Keywords: urban energy modeling, workflow automation, $3 D$ visual editing, Modelica code generation

\section{Introduction}

Building energy modeling (BEM) tools are a key asset in the design of energy efficient buildings. Based on a mathematical model that describes the interaction between a building and its energy system, these tools perform simulations and calculate outputs in terms of energy use and thermal comfort (Hensen and Lamberts 2019).

Recently, the open-source, object-oriented and equation-based modeling language Modelica has become increasingly used in the field of BEM. The use of Modelica has not only grown in the field of BEM, but also in the field of urban building energy modeling (UBEM), where the focus is on the analysis of building stocks and district energy systems. One of the reasons of the increased used of Modelica in BEM and UBEM is that future building and district energy systems integrate multi-domain interconnected subsystems (thermal, hydraulic, electric and control) based on renewable energy generation, for which Modelica provides an appropriate single platform for modeling and simulation. Another reason is that Modelica simulation environments provide a 2D graphical modeling approach that fits well the design of buildings and district energy system topologies. On the other hand, such a 2D graphical approach is inconvenient for modeling the 3D shapes of buildings.

When working on the modeling of a building, Modelica users typically need to abstract the 3D geometry of a building thermal zone into a string of code, which contains area and orientation of the building surfaces. This manual configuration of a thermal building zone is an error-prone process and does not line up with the design workflow of architects and engineers, who are using BIM-based CAD tools such as ArchiCAD or Revit for their 3D building designs. For these reasons, in the last years, different research activities have been focused on the automatic generation of Modelica building energy models from International Foundation Class (IFC) files, which are output of BIM softwares.

Thorade et al. (2015) proposed a toolchain using the commercial simulation tool Simergy. In the first step, the user imports the IFC file as input, adds relevant data with the Simergy graphical user interface (GUI), and then exports the data set as SimModel, which is a data domain model which is able to store information for building energy simulation. In the next step, a mapping tool takes the SimModel file and a Python script generates the Modelica model through a template approach. Reynders et al. (2017) described a tool based on a Python framework. It can read IFC-files, determine the building topology for multi-zone building models, and generate Modelica building models for the Modelica IDEAS library. NytschGeusen et al. (2019) developed an open-source toolchain which can transfer BIM models of 3D building constructions into executable thermal multi-zone Modelica buildings energy models. For this purpose, different opensource libraries and tools were integrated into a Pythonbased software architecture of the toolchain.

In the above mentioned studies, the focus was on the automatic generation of Modelica models for single buildings energy analyses. In terms of urban energy analyses, Remmen et al. (2018) developed TEASER, a Pythonbased automated framework that includes data enrichment, data processing and Modelica model generation for urban context. The tool can produce ready-to-run Modelica building models based on a low order thermal zone model using the IBPSA core library and their larger user libraries Buildings (Wetter et al. 2014), BuildingSystems 
(Nytsch-Geusen et al. 2012), IDEAS (Jorissen et al. 2018) and AixLib (Müller et al. 2016). TEASER can be used with an archetype building approach, which needs only basic input data about a building, but can also use detailed building information for the modeling process. Nevertheless there is no GUI provided for the user, not for parameter settings (e.g. material properties, ventilation rates, etc.) nor for 3D building modeling.

To address the challenges posed by manual translation of 3D building models to Modelica models at urban scale, this paper presents a "Blender-based Automatic Generator of Energy Loads" (BAGEL). BAGEL is an opensource Python-based tool that uses the 3D creation platform Blender as a host application to provide 3D modeling, intuitive GUI and parametrization capabilities for automatic generation of Modelica-based building energy models. The target audience of the tool are scientists and engineers who aim at predicting heating and cooling energy loads of new and existing urban neighborhoods and communities. The paper begins with a description of BAGEL architecture and functionalities, and then proceeds by giving a demonstration of the tool's capabilities with a use case.

\section{Methodology}

This chapter introduces Blender as a host application and then presents the architecture of BAGEL for the conversion of 3D building geometries into Modelica models for urban energy simulations.

\subsection{Blender}

Blender (Blender Online Community 2021) is a free and open-source 3D creation platform. Its functionality includes mesh-based 3D modeling, advanced materials and texture specification, physically based rendering and a Python Application Programming Interface (API) amongst other features. The Python API is deeply integrated allowing, for example, specification of data, control over mesh elements and manipulation of the Blender interface. This enables the development of third-party software (called add-ons in Blender) which function inside Blender and expand its functionality. Some of these features have made Blender an increasingly popular host application for a range of scientific visualisation and analysis tools (Pyka et al. 2010; Scianna 2013; Kent 2013). In the field of building energy simulation, the VI-Suite add-on supports the conversion of geometry and construction materials to the EnergyPlus input format (Southall and Biljecki 2017).

\subsection{BAGEL architecture}

The architecture of BAGEL is shown in Figure 1. Once installed, BAGEL appears as a visual panel directly accessible in the 3D environment of Blender. It consists of three modules:

\section{3D building shaping}

\section{Data enrichment}

3. Modelica model generation

\subsubsection{D building shaping}

In Blender, the geometry of a scene is constructed from one or more objects. These objects can range from basic 3D shapes and lights to illuminate the scene to cameras to take pictures or make video. The relevant object type in the context of BAGEL is the so-called mesh. A mesh is a collection of vertices, edges and faces that describe a $3 \mathrm{D}$ shape. The BAGEL module $3 D$ building shaping allows users to automatically create a new building, which is represented by a 3D mesh whose geometrical and spatial properties can be freely modified using the dedicated visual commands. Such properties are:

- Dimensions along the three axes

- Rotation around the z-axis (i.e. orientation)

\section{- Location in the scene}

BAGEL is currently able to handle only simple building geometries with a low Level of Detail (LoD). The LoD concept is defined in the CityGML standard and defines building models at different levels of complexity and granularity of the geometric representation (Open Geospatial Consortium 2012). In particular, in BAGEL, buildings can only be represented by rectangular prisms, which correspond to LoD1.

\subsubsection{Data enrichment}

At this point, buildings are described in terms of geometrical and spatial design. However, to perform dynamic energy simulations it is necessary to enrich the models with additional properties such as building mass and thermal characteristics of the building envelope. The BAGEL module Data enrichment enables users to define a set of nine properties for each building. This set of properties resembles the input parameters that are needed to solve the resistance-capacitance model described in the ISO 13790 standard (International Standard Organization 2008). This model describes the thermo-physical behavior of buildings by means of an equivalent electric circuit consisting of five resistances and one capacity (5R1C). Such a model has been developed in Modelica language and it will be used as target Modelica model in the next step of the process, where the enriched Blender model will be translated into Modelica code. More details about the 5R1C Modelica model are provided in section 2.2.3

The parameters required to solve the $5 \mathrm{R} 1 \mathrm{C}$ model can be assigned visually to each building in the 3D Blender scene through the BAGEL interface, which stores such parameters within the data structure of the respective building as custom properties. In Blender, custom properties are a way to store metadata in data-blocks 


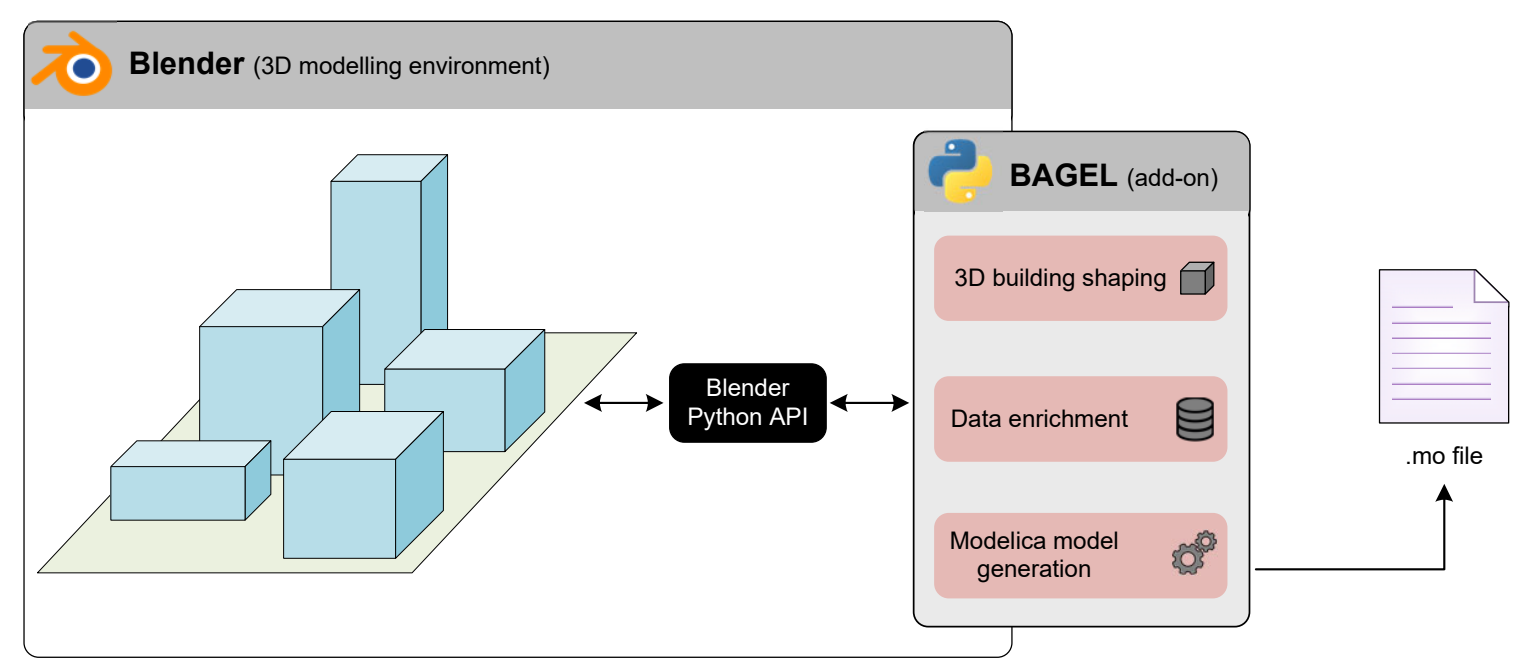

Figure 1. Software architecture of BAGEL.

(e.g. mesh) which can be used by Python scripts to define new settings not available in Blender. The complete list of properties that users need to add and edit through the BAGEL interface is shown in Table 1. In terms of building usage, four different types are available for selection: residential, office, school and hospital. For each building type, BAGEL includes a deterministic schedule defining the amount of internal heat gains and their temporal distribution over a week period. To consider storage effects of the building mass, the thermal heaviness of the building is defined by one of the following options: light, medium and heavy. Each option corresponds to a certain value of the thermal capacitance of the building structure according to the ISO 13790. Regarding thermal characteristics of the building envelope, U-values of walls, floor, roof and windows can be assigned to each building in the Blender scene. In addition, windows have to be defined by a window-to-wall ratio, which represents the ratio of window areas to opaque wall areas, and a solar energy transmission coefficient, which is the ratio of transmitted solar radiation to incident solar radiation. Lastly, users need to provide a value for the air change per hour, which is the measure of how often the air volume is completely changed with outdoor air in one hour.

\subsubsection{Modelica model generation}

Once all the properties have been assigned, BAGEL is able to export Modelica models. Modelica models are stored in simple text files written in the Modelica language. These text files are constructed using a template-based approach (https://www.makotemplates.org/), where placeholders for the necessary simulation parameters are embedded in the file. These necessary parameters are made available by BAGEL and are then mapped to the template automatically. After mapping the parameters defined as placeholders in the Mako template, the last step performed by BAGEL is the rendering of the templates to generate
Table 1. Properties to be assigned to building objects.

\begin{tabular}{lr}
\hline Property & Visual editing method \\
\hline Usage & Multiple choice menu \\
Thermal mass & Multiple choice menu \\
U-value walls & float number \\
U-value roof & float number \\
U-value floor & float number \\
U-value windows & float number \\
Window-to-wall ratio & float number [0:1] \\
G-factor & float number $[0: 1]$ \\
Air change rate & float number \\
\hline
\end{tabular}

the Modelica . mo files.

The code generator was designed for a predefined Modelica model class representing the 5R1C model previously mentioned. This is a lumped-capacitance model where the thermal behavior of the building is described by means of an equivalent resistive-capacitive electrical network consisting of five resistances and one capacitance. Figure 2 shows the scheme of the 5R1C thermal network and its Modelica translation. Depending on the purposes and assumptions, many thermal networks have been proposed in literature, however, the ISO 13790 model is still used for its simplicity, replicability, and few requirements of input parameters. A detailed evaluation of the ISO 13790 model accuracy and limitations can be found in Vivian et al. (2017).

The thermal zone is modeled with three temperature nodes, the indoor air temperature $\left(T_{\text {air }}\right)$, the envelope internal surface temperature $\left(T_{S}\right)$ and the building mass temperature $\left(T_{m}\right)$ and two boundary condition nodes, supply air temperature $\left(T_{\text {sup }}\right)$ and the external air temperature $\left(T_{e}\right)$. The five resistances are related to heat transfer by ventilation $\left(H_{v e}\right)$, windows $\left(H_{t r, w}\right)$, opaque components (split 

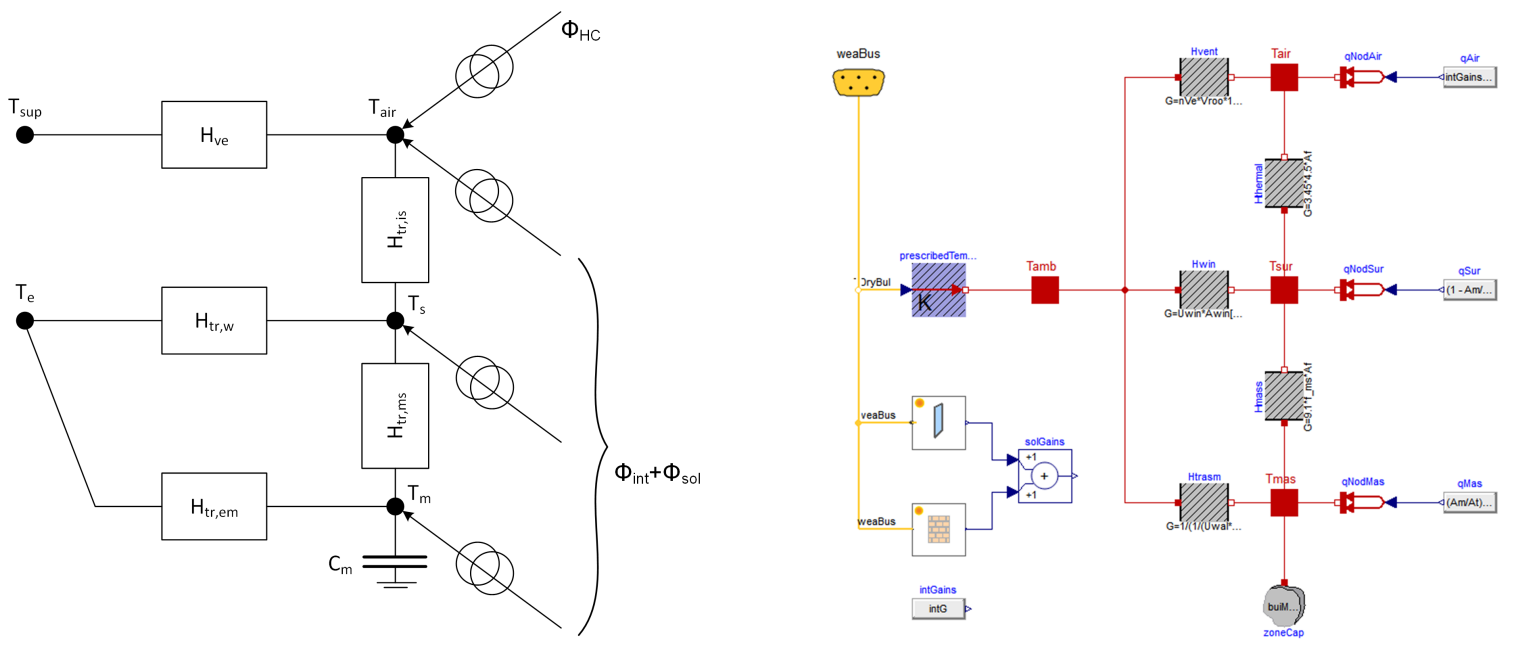

Figure 2. ISO13790 model.

between $H_{t r, e m}$ and $\left.H_{t r, m s}\right)$ and heat transfer between the internal surfaces of walls and the air temperature $\left(H_{t r, i s}\right)$. The thermal capacitance $C_{m}$ is connected with the temperature node $T_{m}$ and includes the thermal capacity of the entire building. The heating and/or cooling demand is found by calculating the heating and/or cooling power $\phi_{H C}$ that needs to be supplied to, or extracted from, the internal air node to maintain a certain minimum or maximum setpoint temperature.

The following code listing demonstrates the principle upon which the Modelica model class is parameterized during the code generation process based on a Mako template.

Listing 1. Text file as Mako template

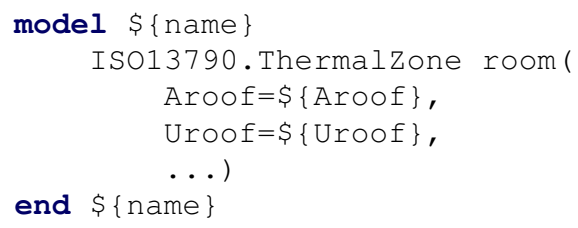

The syntax for the variable substitution is the $\$\{\}$ construct. BAGEL reads the values assigned by the user to the properties of each building in the scene, and then replaces the construct with the correspondent value. As an example, assume that the user created a 3D building model named officel with a roof that has an area of $50 \mathrm{~m}^{2}$ and a U-value of $0.3 \mathrm{~W} / \mathrm{m}^{2} \mathrm{~K}$. BAGEL will generate the following Modelica model, which can be simulated using simulation environments such as Dymola and OpenModelica.

Listing 2. Modelica code generated by BAGEL

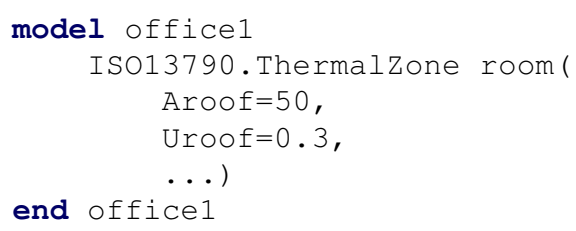

\section{Use case}

To show the capabilities of BAGEL, this chapter presents a use case taken from an ongoing research project. The aim of the project is to carry out a feasibility study of a $5^{\text {th }}$ generation district heating and cooling (5GDHC) system in a new urban area located in the municipality of Køge (Denmark).

5GDHC is a recent technology that combines district heating and cooling supply into a single water network consisting of two pipes (Buffa et al. 2019). Typically, the warm pipe has temperatures of $12-20^{\circ} \mathrm{C}$, while the cold pipe operates in the range $8-16^{\circ} \mathrm{C}$. A particularity of 5 GDHC systems is that they feature a bidirectional distribution, in which the water in each pipe segment can flow in alternating directions, depending on the net thermal fluxes in the system. Bidirectional distribution enables buildings to not only draw, but also feed heat to the network to cover heating demands of other buildings.

The efficiency and profitability of bidirectional 5GDHC systems strongly depends on the heating and cooling demand profiles of the connected buildings and their simultaneity. In particular, the efficiency of 5GDHC systems can be calculated using a metric called Demand Overlap Coefficient (DOC) (Wirtz et al. 2020). For discrete, equally spaced time intervals $t \in T$, the DOC of all buildings $b \in B$ in a district is defined by Equation 1:

$$
D O C=\frac{2 \sum_{t \in T} \min \left\{\sum_{b \in B} \dot{Q}_{h, \text { dem }, b, t}, \sum_{b \in B} \dot{Q}_{c, \text { dem }, b, t}\right\}}{\sum_{t \in T} \sum_{b \in B}\left(\dot{Q}_{h, \text { dem }, b, t}+\dot{Q}_{c, \text { dem }, b, t}\right)}
$$

where $\dot{Q}_{h, d e m}$ is the thermal power demand for heating and $\dot{Q}_{c, \text { dem }}$ is the thermal power demand for cooling. The DOC ranges between 0 and 1 . A DOC of 0 means that heating and cooling demand profiles do not overlap at all, a DOC of 1 means they match exactly. It can be noted that the District DOC is calculated solely on the basis of building energy demands.

In this context, the tool BAGEL was used to create a 

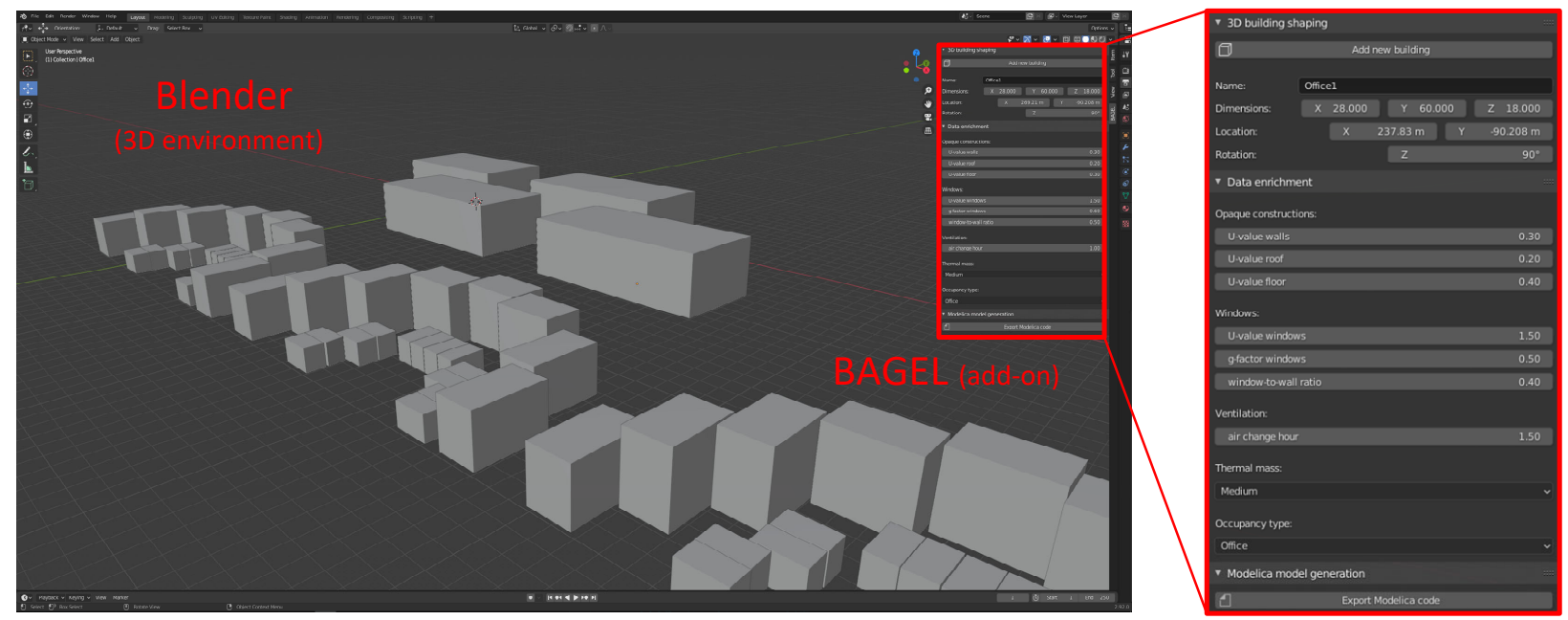

Figure 3. Blender interface with BAGEL add-on

3D model of the new urban area in Køge, visually enrich the building models with relevant properties, and export ready-to-run Modelica models. Figure 3 shows the 3D model in the Blender interface with the BAGEL add-on.

The portion of urban area considered in this use case is planned to be constructed during the next years and it includes a mix of residential and commercial buildings, as illustrated in Table 2. The required geometrical and thermal properties were assigned to the $3 \mathrm{D}$ building models using the BAGEL visual panel. Since this is a new urban area, and not all building information are available yet, properties were estimated according to architectural master plan, Danish building regulations and authors' assumptions. Once all properties had been assigned, Modelica files were exported by pressing the button "Export Modelica code". Then, hourly simulations were performed using Dymola. Exporting time was about $0.01 \mathrm{~s}$ per building, while simulation time was about $2 \mathrm{~s}$ per building for an one-year period. Simulations were carried out on a laptop PC with a $1.6 \mathrm{GHz}$ CPU. Note that the actual location of the buildings in the scene does not affect simulation results, as buildings are completely independent from each other. Factors such as heat island effect and shading between buildings are not considered.

Table 2. Buildings details.

\begin{tabular}{lrr}
\hline Typology & No. buildings & Total floor area \\
\hline Single-family house & 48 & $4320 \mathrm{~m}^{2}$ \\
Block apartment & 32 & $25600 \mathrm{~m}^{2}$ \\
Office & 4 & $36000 \mathrm{~m}^{2}$ \\
\hline
\end{tabular}

Figure 4 shows the total heating and cooling demand profiles of the urban area over an one-year period. Heating demand for domestic hot water and cooling demand for server rooms in offices were added in post-processing as constant profiles. Hourly values of total heating and cooling demand were used to calculate the DOC of the urban

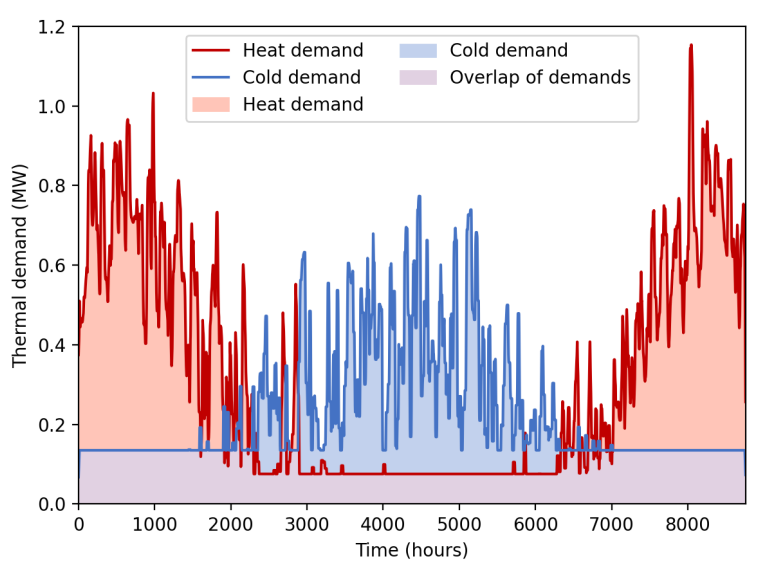

Figure 4. Heating and cooling demand profiles (daily average).

area by applying Equation 1 . The calculation resulted in a DOC equals to 0.38. According to Wirtz et al. (2020), for DOCs larger than 0.3 , a heating and cooling supply with a 5GDHC system has a higher exergy efficiency compared to a reference system. Since the calculation of the DOC is based only on heating and cooling building demand, no models related to the district energy network were needed in this case study. The actual modeling and simulation of the district energy network is planned as a next step during the project.

\section{Conclusion and outlook}

This paper presented the development of BAGEL, an open-source tool that provides automatic conversion of 3D building models into Modelica models for urban energy simulations. The software is written in Python programming language and it is developed as an add-on for the 3D creation suite Blender. BAGEL enables users to create 3D building geometries, perform data enrichment and execute generation of ready-to-run low order Modelica building models. All these actions can be performed graphically 
through the BAGEL interface, which is embedded in the Blender 3D environment as a visual panel. The possibility to rapidly create $3 \mathrm{D}$ building geometries from scratch can be particularly useful in early design stages of new urban districts and neighborhoods, when CityGML and BIM files are not usually available.

To demonstrate the capabilities of BAGEL, a use case was introduced. A 3D enriched model of a new urban area in Denmark was developed in order to calculate heating and cooling demand. This allowed to estimate the efficiency and profitability of a 5GHC system by simulating the generated Modelica models in Dymola.

Future developments of BAGEL will focus on the integration of archetype building models through the software TEASER. In addition, possibilities to account for mutual shading between buildings will be investigated. Moreover, visualization of simulation results within the Blender scene will be explored. The source code of BAGEL, together with the Modelica ISO 13790 model, will be released on GitHub by the end of October 2021.

\section{Acknowledgements}

This work was financially supported by the Danish Energy Agency, under the Energy Technology Development and Demonstration Program (EUDP).

We gratefully acknowledge the financial support by Federal Ministry for Economic Affairs and Energy (BMWi), promotional reference 03EWR020E (Reallabor der Energiewende: TransUrban.NRW).

This work emerged from the IBPSA Project 1, an international project conducted under the umbrella of the International Building Performance Simulation Association (IBPSA). Project 1 will develop and demonstrate a BIM/GIS and Modelica Framework for building and community energy system design and operation.

\section{References}

Blender Online Community (2021). Blender - a 3D modelling and rendering package. Blender Foundation. Blender Institute, Amsterdam. URL: http://www.blender.org.

Buffa, Simone et al. (2019). "5th generation district heating and cooling systems: A review of existing cases in Europe". In: Renewable and Sustainable Energy Reviews 104, pp. 504522. DOI: 10.1016/j.rser.2018.12.059.

Hensen, Jan and Roberto Lamberts (2019). Building Performance Simulation for Design and Operation. Routledge. ISBN: 9781138392199.

International Standard Organization (2008). ISO 13790:2008 Energy performance of buildings - Calculation of energy use for space heating and cooling.

Jorissen, Filip et al. (2018). "Implementation and Verification of the IDEAS Building Energy Simulation Library". In: Journal of Building Performance Simulation 11 (6), pp. 669-688. DOI: 10.1080/19401493.2018.1428361.

Kent, Brian R. (2013). "Visualizing Astronomical Data with Blender". In: Publications of the Astronomical Society of the Pacific 125.928, pp. 731-748. DOI: 10.1086/671412.
Müller, Dirk et al. (2016-09). “AixLib - An Open-Source Modelica Library within the IEA-EBC Annex 60 Framework". In: BauSIM2016 Conference.

Nytsch-Geusen, Christoph et al. (2012-09). "Modelica BuildingSystems - Eine Modellbibliothek zur Simulation komplexer energietechnischer Gebäudesysteme". In: BauSIM2012 Conference.

Nytsch-Geusen, Christoph et al. (2019-03). "BIM2Modelica An open source toolchain for generating and simulating thermal multi-zone building models by using structured data from BIM models". In: 13th International Modelica Conference, pp. 33-39. DOI: 10.3384/ecp1915733.

Open Geospatial Consortium (2012). OGC city geography markup language (CityGML) encoding standard 2.0.0. Tech. rep.

Pyka, Martin et al. (2010). "fMRI data visualization with BrainBlend and Blender". In: Neuroinformatics 8.1, pp. 21-23. DOI: 10.1007/s12021-009-9060-3.

Remmen, Peter et al. (2018). "TEASER: an open tool for urban energy modelling of building stocks". In: Journal of Building Performance Simulation 11.1, pp. 84-98. DOI: 10.1080/ 19401493.2017.1283539.

Reynders, Glenn et al. (2017-08). "Towards an IFC-Modelica tool facilitating model complexity selection for building energy simulation". In: 15th IBPSA Conference, pp. 22572266.

Scianna, Andrea (2013). "Building 3D GIS data models using open source software". In: Applied Geomatics 5.2, pp. 119132. DOI: $10.1007 / \mathrm{s} 12518-013-0099-3$.

Southall, Ryan and Filip Biljecki (2017). "The VI-Suite: a set of environmental analysis tools with geospatial data applications". In: Open Geospatial Data, Software and Standards 2.23, pp. 1-13. DOI: 10.1186/s40965-017-0036-1.

Thorade, Matthis et al. (2015-09). "An open toolchain for generating Modelica code from Building Information Models". In: 11th International Modelica Conference, pp. 383-391. DOI: 10.3384/ecp15118383.

Vivian, Jacopo et al. (2017). "An evaluation of the suitability of lumped-capacitance models in calculating energy needs and thermal behaviour of buildings". In: Energy and Buildings 150, pp. 447-465. ISSN: 0378-7788. DOI: https ://doi .org/ 10.1016/j.enbuild.2017.06.021.

Wetter, Michael et al. (2014). "Modelica Buildings library". In: Journal of Building Performance Simulation 7.4, pp. 253270. DOI: 10.1080/19401493.2013.765506.

Wirtz, Marco et al. (2020). "Quantifying demand balancing in bidirectional low temperature networks". In: Energy and Buildings 224. DOI: 10.1016/j.enbuild.2020.110245. 